See Article page 13 .

\section{Commentary: How do you size a frozen elephant trunk?}

\author{
Roland Assi, MD, MMS, and Arnar Geirsson, MD
}

Kandola and colleagues ${ }^{1}$ report their single-center experience with the frozen elephant trunk (FET) procedure for thoracic aortic aneurysms. They specifically look into patients who were intended to have single-stage procedures, presumably for aneurysms limited to the aortic arch and proximal descending thoracic aorta. They identified 36 patients over a period of 11 years (2008-2019). The endoleak or sac expansion rate during the follow-up period (mean of 2 years) was $36 \%$, with the vast majority of cases being identified on the first postoperative scan. A careful review of preoperative and postoperative aortic measurements revealed that the vast majority of patients who had an endoleak/sac expansion had less than $10 \%$ FET oversizing and/or less than 30-mm distal seal zone. None of the patients who had more than $10 \%$ oversizing and more than 30-mm distal seal zone experienced endoleak/sac expansion. Based on this data, they recommended more than $10 \%$ oversizing and more than $30-\mathrm{mm}$ seal zone for FET procedures performed for thoracic aortic aneurysms. Of note, their center's rate of paraplegia with FET was $1 \%$, but none of the patients included was affected.

The findings are not surprising, given the known association between undersizing or insufficient seal zone and endoleak. However, this raises the question about the optimal sizing criteria for FET implantation for aneurysms. Data could be extrapolated from the thoracic endovascular aortic repair experience on the minimal

\footnotetext{
From the Division of Cardiac Surgery, Department of Surgery, Yale University School of Medicine, New Haven, Conn.

Disclosures: Dr Assi reported no conflicts of interest. Dr Geirsson reported member of Medtronic's Strategic Surgical Advisory Board.

The Journal policy requires editors and reviewers to disclose conflicts of interest and to decline handling or reviewing manuscripts for which they may have a conflict of interest. The editors and reviewers of this article have no conflicts of interest.

Received for publication June 6, 2020; revisions received June 6, 2020; accepted for publication June 10, 2020; available ahead of print June 20, 2020.

Address for reprints: Arnar Geirsson, MD, 330 Cedar St, New Haven, CT 06519 (E-mail: arnar.geirsson@yale.edu).

JTCVS Techniques 2020;3:21-2

2666-2507

Published by Elsevier Inc. on behalf of The American Association for Thoracic Surgery. This is an open access article under the CC BY-NC-ND license (http:// creativecommons.org/licenses/by-nc-nd/4.0/).

https://doi.org/10.1016/j.xjtc.2020.06.013
}

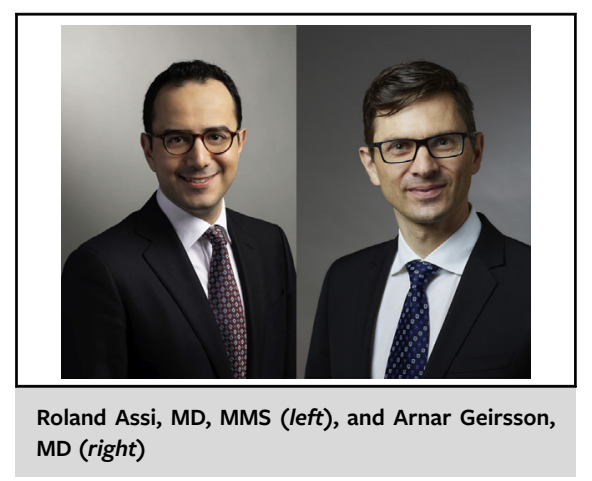

CENTRAL MESSAGE

The optimal sizing criteria for

frozen elephant trunk proced-

ures for aortic aneurysms are not

well known. The surgeon should

weigh the risk of endoleak versus

aortic wall injury and paraplegia.

recommended criteria for implantation (in general oversize $>10 \%$ and seal zone $>20 \mathrm{~mm}$ ); however, we do not have a large registry of FET procedures for aortic aneurysms that addresses this question. In addition, as the authors allude to, some FET devices recommend more aggressive criteria (>15\%-25\% oversizing, $>40-\mathrm{mm}$ seal zone).

Oversizing is desired to prevent stent-migration and endoleak. However, aggressive oversizing is not without consequences. We know that aggressive oversizing is associated with the risk of distal aortic injury such as rupture and stent-induced new entry tears. Similarly, extensive aortic length coverage may improve the seal zone but at the cost of increased risk of paraplegia. In this sense, conservative oversizing followed by a staged thoracic endovascular aortic repair may represent a safer alternative. In addition, one should not expect the same sizing criteria to be applicable to all devices; different stent-grafts may have different mechanistic relationships to the aortic wall depending on the material used and the device's design.

Recently, certain hybrid FET devices were granted permission to the US market. This will likely result in an increased use of this technology for thoracic aortic aneurysms. It is imperative that aortic centers join efforts to report and analyze their outcomes, 
which would generate enough evidence to guide the sizing of FET. Until then, the sizing criteria proposed by the authors appear to be reasonable and safe.

\section{Reference}

1. Kandola S, Abdulsala A, Field M, Fisher RK. Frozen elephant trunk repair of aortic aneurysms: how to reduce the incidence of endoleak and reintervention. J Thorac Cardiovasc Surg Tech. 2020;3:13-20. 